\title{
Detection and Evaluation of Adverse Drug Reaction Signals of Antidepressants Based on FDA Adverse Event Reporting System Database
}

\author{
Chao Ran, Hu Zhou, Chao Tan, Juntao Tan, Zhengyu Zhang, Wenlong Zhao* \\ School of Medical Informatics of Chong Qing Medical University, Chongqing, China \\ Email: *cqzhaowl@163.com
}

How to cite this paper: Ran, C., Zhou, H., Tan, C., Tan, J. T., Zhang, Z. Y., \& Zhao, W. L. (2020). Detection and Evaluation of Adverse Drug Reaction Signals of Antidepressants Based on FDA Adverse Event Reporting System Database. Open Journal of Depression, 9, 17-25.

https://doi.org/10.4236/ojd.2020.92002

Received: April 23, 2020

Accepted: May 24, 2020

Published: May 27, 2020

Copyright $\odot 2020$ by author(s) and Scientific Research Publishing Inc. This work is licensed under the Creative Commons Attribution International License (CC BY 4.0).

http://creativecommons.org/licenses/by/4.0/

(c) (i) Open Access

\begin{abstract}
Background: Pre-marketing clinical research of drugs can not completely solve the safety problems in the process of wide application of drugs post-marketing, so it is necessary to re-evaluate the safety and effectiveness of drugs after marketing. Objective: To detect and analyze the adverse drug reaction (ADR) signals of Selective Serotonin Reuptake Inhibitor (SSRIs) post-marketing and provide references for clinical rational drug use. Methods: Reporting Ratio (ROR) method was used to mine the adverse reaction signals of SSRIs in the Adverse Reaction Reporting System (ARES) of the Food and Drug Administration of the United States (FDA), and the results were analyzed and evaluated. Results: Adverse Drug Events (ADEs) of fluoxetine, fluvoxamine, paroxetine, sertraline and citalopram were 40,217, 2907, 52,439, 63,849 and 42,588 cases respectively. After ROR test, there were 187 ADR signals of the five drugs, among which ADR was most prominent in psychiatric and nervous system. It mainly includes adverse reactions such as anxiety, depression, suicidal ideation, 5-HT syndrome, withdrawal syndrome and so on. Conclusion: The study based on ADR signals in the real world is helpful to evaluate the post-marking safety drugs and provide references for safety in clinical medication.
\end{abstract}

\section{Keywords}

Antidepressants, SSRIs, Adverse Drug Reactions, Data Mining, Signal Detection

\section{Introduction}

As a widely used drug in clinic, Selective Serotonin Reuptake Inhibitors (SSRIs) can selectively inhibit the recovery of 5-hydroxytryptamine (5-HT) by presynaptic membrane, improve the depressive symptoms and cognitive function of pa- 
tients (He et al., 2018). The currently used SSRIs mainly include Fluoxetine, Paroxetine, Sertraline, Fluvoxamine and Citalopram. Although antidepressants have the advantages of high selectivity for 5-HT receptors, little impact on other neurotransmitters, long half-life and good patient compliance, the incidence of side effects during treatment is still as high as $31 \%-60 \%$ ( $\mathrm{Zhu}, 2018$ ). The adverse event reporting system database established by the U.S. Food and drug administration is the world's second largest spontaneous adverse event reporting database after the VigiBase database established by the World Health Organization. Up to now, more than 10 million adverse event reports have been received from all over the world. Since 2004, FDA has provided the public with open source downloads of adverse event reports it receives on a quarterly basis. In this study, ADR risk signal mining of SSRIs drugs was carried out by using FAERS database to provide reference for clinical rational drug use.

\section{Materials and Methods}

\subsection{Data Sources}

The data comes from the Food and Drug Administration (FDA) Adverse Event Reporting System (FAERS). In this study, ADR reports from January 1, 2004 to December 31, 2018 were included and tested.

\subsection{Data Preprocessing}

In this study, the data was extracted by using the OpenVigilFDA analysis tool (http://openvigil.sourceforge.net), which directly obtained the original information in the FAERS database through the API of application program interface and processed it in a structured way, with high extraction efficiency and accuracy The extraction efficiency is high and accurate (Böhm et al., 2016). According to the World Health Organization (WHO) Anatomical Therapeutic Chemical and the Defined Daily Dose (ATC/DDD), drug dictionary to obtain the specific drug specification names of SSRIs, including five: Fluoxetine, Paroxetine, Sertraline, Fluvoxamine and Citalopram. By searching the above five standardized English names of drugs and the role of adverse drug reactions as the primary suspicion "PS (Primary Suspect Drug)", a report was obtained that the primary suspected drugs were SSRIs.

\subsection{Signal Detection Method}

Disproportionality method Analysis (DPA) is a common method for data mining of adverse drug reactions. In this study, DPA is used as a signal monitoring method for adverse drug reactions. This method is based on the four grid table of drug adverse event (shown in Table 1). If the number of a drug associated with an adverse event is greater than the expected number, it is called a proportion imbalance, indicating that the drug and the adverse event are related The probability of the event appearing in one report at the same time is greater than that of other drugs and other adverse events appearing in one report at the same 
Table 1. Two-by-two contingency table of DPA.

\begin{tabular}{ccc}
\hline & $\begin{array}{c}\text { Reports with the suspected } \\
\text { ADR }\end{array}$ & $\begin{array}{c}\text { Reports without the } \\
\text { suspected ADR }\end{array}$ \\
\hline Reports with the suspected drug & $\mathrm{a}$ & $\mathrm{b}$ \\
Reports without the suspected drug & $\mathrm{c}$ & $\mathrm{d}$ \\
\hline
\end{tabular}

time. It can be considered that there is a potential connection between the drug and the adverse event, which may be a drug adverse reaction signal. According to different statistical principles, DPA can be further divided into proportional reporting ratio (PRR), reporting odds ratio (ROR) and Bayesian confidence propagation neural network (BCPNN), and so on. The measurement table of ratio imbalance is shown in Table 1.

The ROR method has a high sensitivity and a good ability to detect ADR signals in the early stage, so this method was adopted in this study (Rothman et al., 2004). The reporting odds ratio (ROR) can be expressed as

$$
R O R=\frac{(a / c)}{(b / d)}=\frac{a d}{b c} .
$$

The standard error of $\ln (R O R)$ and $95 \%$ confidence interval can be calculated by

$$
\begin{gathered}
S E(\ln R O R)=\sqrt{\left(\frac{1}{\mathrm{a}}+\frac{1}{b}+\frac{1}{c}+\frac{1}{d}\right)} . \\
95 \% C I=\mathrm{e}^{\operatorname{In}(R O R) \pm 1.96 S E(\ln R O R)} .
\end{gathered}
$$

When $a \geq 3$, i.e. SSRIs drug suspected adverse event report is greater than or equal to 3 , and the lower limit of $95 \%$ CI of ROR is greater than 1, it indicates the production of a drug adverse reaction signal.

\subsection{Signal Classification}

FAERS database uses the preferred terms (PT) of the medical dictionary for regulatory activities (MedDRA) to code the adverse drug reaction (ADR), and to structurally process the original data (Wong et al., 2015). All terms in MedDRA are given a unique code and classified into five levels: systemic-organ classification, High Level Group Term (HLGT), High Level Term (HLT), prime Term (PT) and Lowest Level Term (LLT). The basic unit is the PT, which is used to classify and retrieve medical events. MedDRA is not only used for the standardized processing and coding of adverse drug events, but also provides classification information of adverse drug events (Yu, 2016). In this study, the excavated ADR signals were statistically sorted according to the system organ class (SOC) of MedDRA 19.0.

\section{Result}

\subsection{Basic Result}

By retrieving the FAERS data from January 1, 2014 to December 31, 2018, events 
involving uncertain names of food, medical devices, and drugs were removed, and a total of 6,727,779 ADR reports were obtained. The ADEs with Fluoxetine, Fluvoxamine, Paroxetine, Sertraline and Citalopram as the primary suspects were 40,217, 2907, 52,439, 63,849 and 42,588 cases, respectively. The ADR signals calculated by ROR method were 1583, 429, 1481, 1831 and 1459 cases, respectively, among which 187 cases showed ADR signals in all five drugs. The ADR caused by SSRI drugs mainly include psychiatric disorders, nervous system disorders, congenital, familial and genetic disorders and investigations (for example, abnormality of various laboratory and physical inspection indexes).

\subsection{Hierarchical Mining Results of SSRI Drug-SOC Classification Combination}

The ROR algorithm was used to mine ADR signals of SSRIs drugs, and the queried ADR signals were measured SOC classification referring to the MedDRA terminology. The heatmap was drawn to visually display the excavated ADR signals, as shown in Figure 1. It can be seen from the clustering tree on the upper side of the figure that there were more ADR signals in fluoxetine, paroxetine, sertraline and citalopram, and the ADR signals cover all classification of systemic organs while there was less adverse reaction signal in fluvoxamine, and adverse reaction signal covers the classification system of organs including the psychiatric disorders, nervous system disorders, congenital, familial and genetic disorders and investigations.

Figure Labels: This figure shows the distribution of adverse drug reaction signals. The horizontal axis represents the type of SSRIs drug, the vertical axis represents the SOC classification of adverse events, and each cell at the intersection of rows and columns represents the combination of SSRIs drugs and SOC
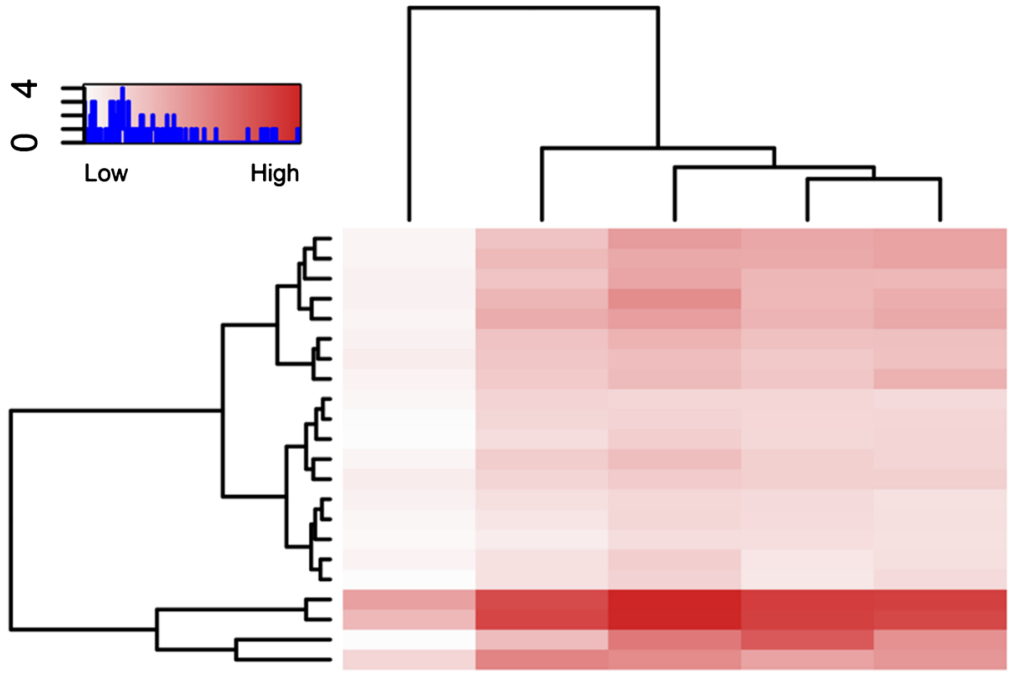

Musculoskeletal and connective tissue disorders Respiratory thoracic and mediastinal disorders Gastrointestinal disorders Vascular disorders Injury, poisoning and procedural complications Metabolism and nutrition disorders Pregnancy, puerperium and perinatal conditions Renal and urinary disorders

Neoplasms benign malignant and unspecified Reproductive system and breast disorders General disorders and administration site condition Skin and subcutaneous tissue disorders Endocrine disorders Social circums Surgical and medical procedures Psychiatric disorders disorders Congenital, familial and genetic disorders

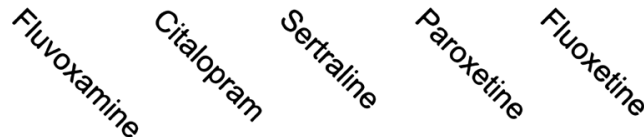

Figure 1. ADR signal heatmap of SSRIs-SOC classification hierarchy. 
classification. The color of each cell represents the frequency of the adverse reaction signal. The darker the color, the greater the frequency. White indicates that the "drug-SOC classification combination" did not detect an adverse drug reaction signal.

The hot spot map shows the results of cluster analysis of adverse reaction signals from two dimensions: SSRIs category and adverse event SOC. First, it can be seen from the cluster tree on the left of the figure that it can be divided into two major categories: 1) Fluoxetine, Paroxetine, Sertraline, and Citalopram cover all different levels of adverse events, so its clusters are under the same cluster; 2) Fluvoxamine has fewer adverse reaction signals and is clustered into a cluster. Compared with Fluoxetine and Paroxetine, Sertraline and Citalopram have relatively few adverse reaction signals, so the four drug varieties are further divided. From the cluster tree at the top of the figure, it can be seen that the signals of adverse drug reactions detected by the five SSRIs are mostly concentrated in 4 SOC classifications including various psychiatric disorders, various nervous system disorders, various congenital, familial and genetic disorders and various investigations, among which Fluoxetine detected as many as 1853 danger signals, Fluvoxamine has the fewest danger signals, only 429 . The positive adverse drug reaction signals of the four systems are shown in Table 2.

Table 2. Signal detection results of SSRIs in key systems.

\begin{tabular}{|c|c|c|c|c|c|}
\hline & Fluvoxamine & Fluoxetine & Paroxetine & Sertraline & Citalopram \\
\hline \multicolumn{6}{|l|}{ Anxiety } \\
\hline $\mathrm{N}$ & 195 & 2164 & 4216 & 3930 & 2097 \\
\hline ROR & 4.00 & 3.20 & 5.00 & 3.74 & 2.92 \\
\hline $95 \% \mathrm{CI}$ & $(3.46,4.63)$ & $(3.07,3.35)$ & $(4.85,5.17)$ & $(3.62,3.87)$ & $(2.79,3.05)$ \\
\hline \multicolumn{6}{|c|}{ Depression } \\
\hline $\mathrm{N}$ & 134 & 2623 & 3154 & 3634 & 1997 \\
\hline ROR & 3.09 & 4.56 & 4.20 & 3.97 & 3.19 \\
\hline $95 \% \mathrm{CI}$ & $(2.61,3.70)$ & $(4.38,4.75)$ & $(4.05,4.35)$ & $(3.84,4.11)$ & $(3.05,3.34)$ \\
\hline \multicolumn{6}{|c|}{ Withdrawal syndrome } \\
\hline $\mathrm{N}$ & 54 & 634 & 7604 & 859 & 583 \\
\hline ROR & 3.87 & 3.26 & 44.82 & 2.83 & 2.87 \\
\hline $95 \% \mathrm{CI}$ & $(2.96,5.07)$ & $(3.01,3.53)$ & $(43.61,46.06)$ & $(2.65,3.03)$ & $(2.64,3.12)$ \\
\hline \multicolumn{6}{|c|}{ Sleep disorder } \\
\hline $\mathrm{N}$ & 114 & 1426 & 2753 & 2776 & 1387 \\
\hline ROR & 2.86 & 2.67 & 3.97 & 3.24 & 2.38 \\
\hline $95 \% \mathrm{CI}$ & $(2.37,3.45)$ & $(2.53,2.81)$ & $(3.81,4.13)$ & $(3.12,3.37)$ & $(2.25,2.51)$ \\
\hline \multicolumn{6}{|c|}{ Suicidal ideation } \\
\hline $\mathrm{N}$ & 110 & 1596 & 3577 & 2194 & 1239 \\
\hline ROR & 5.87 & 6.34 & 11.76 & 5.52 & 4.56 \\
\hline $95 \% \mathrm{CI}$ & $(4.85,7.10)$ & $(6.03,6.67)$ & $(11.35,12.18)$ & $(5.28,5.76)$ & $(4.31,4.83)$ \\
\hline
\end{tabular}




\section{Continued}

\begin{tabular}{|c|c|c|c|c|c|}
\hline Drowsines & & & & & \\
\hline $\mathrm{N}$ & 110 & 946 & 1421 & 1164 & 1164 \\
\hline ROR & 4.16 & 2.56 & 2.98 & 2.54 & 2.99 \\
\hline $95 \%$ CI & $(3.43,5.02)$ & $(2.40,2.73)$ & $(2.82,3.14)$ & $(2.41,2.67)$ & $(2.82,3.18)$ \\
\hline \multicolumn{6}{|c|}{ Irritability } \\
\hline $\mathrm{N}$ & 112 & 894 & 2188 & 1277 & 966 \\
\hline ROR & 8.27 & 4.78 & 2.98 & 4.33 & 4.89 \\
\hline $95 \%$ CI & $(6.84,9.99)$ & $(4.47,5.11)$ & $(2.82,3.14)$ & $(4.09,4.58)$ & $(4.58,5.22)$ \\
\hline \multicolumn{6}{|l|}{ Tremor } \\
\hline $\mathrm{N}$ & 124 & 1112 & 2453 & 1920 & 1283 \\
\hline ROR & 5.02 & 3.24 & 5.17 & 3.57 & 3.55 \\
\hline $95 \%$ CI & $(4.19,6.00)$ & $(3.05,3.44)$ & $(5.48,5.95)$ & $(3.41,3.74)$ & $(3.35,3.75)$ \\
\hline \multicolumn{6}{|l|}{ Amnesia } \\
\hline $\mathrm{N}$ & 40 & 441 & 756 & 718 & 444 \\
\hline ROR & 3.31 & 2.66 & 3.54 & 2.77 & 2.51 \\
\hline $95 \% \mathrm{CI}$ & $(2.43,4.53)$ & $(2.42,2.92)$ & $(3.29,3.81)$ & $(2.57,2.98)$ & $(2.29,2.75)$ \\
\hline \multicolumn{6}{|c|}{ 5-HT syndrome } \\
\hline $\mathrm{N}$ & 95 & 692 & 662 & 956 & 706 \\
\hline ROR & 37.00 & 21.10 & 14.31 & 19.25 & 20.44 \\
\hline $95 \% \mathrm{CI}$ & $(30.18,45.57)$ & $(19.56,22.94)$ & $(13.17,1.56)$ & $(17.96,20.63)$ & $(18.89,22.12)$ \\
\hline \multicolumn{6}{|c|}{ Dyskinesia } \\
\hline $\mathrm{N}$ & 27 & 307 & 415 & 497 & 299 \\
\hline ROR & 4.27 & 3.55 & 3.70 & 3.66 & 3.26 \\
\hline $95 \% \mathrm{CI}$ & $(2.92,6.24)$ & $(3.17,3.98)$ & $(3.36,4.08)$ & $(3.34,4.00)$ & $(2.91,3.66)$ \\
\hline \multicolumn{6}{|c|}{ Weight increased } \\
\hline $\mathrm{N}$ & 87 & 1237 & 2019 & 1749 & 950 \\
\hline ROR & 2.92 & 3.04 & 3.86 & 2.70 & 2.17 \\
\hline $95 \% \mathrm{CI}$ & $(2.36,3.61)$ & $(2.87,3.21)$ & $(3.69,4.04)$ & $(2.58,2.84)$ & $(4.58,5.22)$ \\
\hline \multicolumn{6}{|c|}{ Cholesterolemia } \\
\hline $\mathrm{N}$ & 25 & 455 & 434 & 749 & 370 \\
\hline ROR & 2.90 & 3.89 & 2.83 & 4.08 & 2.96 \\
\hline $95 \% \mathrm{CI}$ & $(1.95,4.30)$ & $(3.54,4.27)$ & $(2.57,3.11)$ & $(3.79,4.39)$ & $(2.67,3.28)$ \\
\hline \multicolumn{6}{|c|}{ Blood triglycerides increased } \\
\hline $\mathrm{N}$ & 22 & 243 & 434 & 314 & 204 \\
\hline ROR & 5.69 & 4.62 & 2.83 & 3.77 & 3.64 \\
\hline $95 \% \mathrm{CI}$ & $(3.74,8.65)$ & $(4.07,5.25)$ & $(2.57,3.11)$ & $(3.37,4.23)$ & $(3.17,4.19)$ \\
\hline \multicolumn{6}{|c|}{ Neutrophilia } \\
\hline $\mathrm{N}$ & 13 & 71 & 91 & 108 & 64 \\
\hline ROR & 9.06 & 3.61 & 3.56 & 3.48 & 3.06 \\
\hline $95 \%$ CI & $(5.25,15.63)$ & $(2.85,4.57)$ & $(2.89,4.39)$ & $(2.88,4.22)$ & $(2.39,3.92)$ \\
\hline
\end{tabular}

Note: $\mathrm{N}$ represents the number of ADRs in the drug. 


\section{Discussion}

In this study, the FAERS database was used to mine the ADR risk signals of SSRIs drugs, focusing on the ADR risk signals of Fluoxetine, Fluvoxamine, Paroxetine, Sertraline and Citalopram in various psychiatric disorders, nervous system disorders and investigations ADRs of various mental illness systems are the most dangerous signals that have been discovered this time, mainly including anxiety, depression, insomnia, irritability, withdrawal syndrome, and suicidal tendencies. The reason may be related to the decrease of 5-HT receptor sensitivity in brain caused by long-term SSRIs administration, and the decrease of 5 -HT concentration in synaptic space caused by sudden discontinuation of SSRIs administration, thus leading to the occurrence of the above symptoms (Luo et al., 2008). Among them, suicidal tendency was the most dangerous signal among all kinds of mental system diseases. In 2004, the FDA conducted a comprehensive meta-analysis of the effects of antidepressants on suicide risk in pediatric $(<18$ years old) patients, and risk assessment analysis showed that antidepressants were associated with an increased risk of suicide. Based on the results of this meta-analysis, the FDA in 2004 increased the black box warning of increased risk of suicide in minors ( $<18$ years old) after medication, especially when starting treatment and changing drug dosage (Hammad, Laughren, \& Racoosin, 2006). After further study and analysis, the scope of black box warning was extended to children and adolescents under 24 years old in 2007 (Leon, 2007; Friedman \& Leon, 2007). In the treatment of depression, the US Primary Healthcare Guidelines recommend that adolescents with mild depression should be actively supported and monitored. And for patients with moderate and severe depression, SSRIs can be used (Radovic et al., 2014; Nardi et al., 2013). Therefore, when SSRIs are used to treat patients with depression, the severity of the patient's condition should be fully evaluated and the patient's behavioral tendency should be paid close attention to.

The common ADR signals of nervous system include dizziness, extrapyramidal reaction, 5-HT syndrome and epilepsy (Zhou \& Chen, 2010). Among them, our study found that the main clinical manifestations of 5-HT syndrome were symptoms of anxiety, irritability, myoclonus, blurred consciousness, and agitation, which generally appear after the combination drug treatment or increase of the dosage. Therefore, the dosage can be timely adjusted according to individual symptoms in the treatment process. For patients with acute phase, after increasing the dosage to stabilize the condition, the dose should be timely adjusted back to the range of maintenance treatment. In this study, in addition to the common neurological adverse reactions described above, signals of Amnesia and Dyskinesia were also detected.

Other adverse reactions include sinus arrhythmia, weight gain, and neutrophils. Although SSRIs have mild side effects in the cardiovascular system and other systems, a report based on the safety monitoring of antidepressants from 1993 to 2010 shows that SSRIs have an increased risk of arrhythmias by inducing 
longer QTc intervals (Spindelegger et al., 2015). Weight gain may be caused by the improvement of depression or other reactions of depression, resulting in eating more and sleeping more, and then weight gain (Wang, 2011). In addition, it has been reported that the use of antidepressants can cause leukopenia $(\mathrm{Ng}$, Kennar, \& Uetrecht, 2014). Therefore, in the clinical application of antidepressants, patients' white blood cell levels should be checked regularly to identify risks and symptomatic treatment in a timely manner.

In this study, the signal mining of SSRIs adverse drug reaction events in the FAERS database in the United States revealed the potential association between many adverse events and SSRIs drugs, which provided the research hypothesis for subsequent studies in multiple disciplines including clinical, pharmaceutical, epidemiological and other disciplines. It also lays the foundation for pharmacovigilance surveillance based on "real world data". Compared to the traditional drug safety evaluation methods such as pre-market clinical randomized controlled trials (randomized controlled trials, RCT), etc., with a large sample size and long span cycle, wide coverage, etc., can effectively reflect the SSRIs drug adverse reaction caused by distribution in the real world, can be used as a traditional medicine extension and supplement of safety evaluation method. However, this study has some limitations. First, studies based on adverse event reports could not calculate the incidence of each adverse event because they could not obtain information about the drug population. Secondly, this study is a retrospective observational study. The ADR signals obtained by data mining cannot be defined as causality at will, which requires further assessment and verification by experts in related fields.

\section{Conclusion}

In this study, data mining technology was used to mine 6727779 ADR reports in the FEARS database. It was found that the ADR signals of five SSRIs mainly included nervous system damage, mental system damage and laboratory examination abnormality, including 1583 ADR signals of Fluoxetine, 429 ADR signals of Fluvoxamine, 1481 ADR signals of Paroxetine. There were 1831 adverse reaction signals of Sertraline and 1459 adverse reaction signals of Citalopram. In addition, the adverse reaction signals of SSRIs discovered in this study included suicidal tendency, which suggested that when SSRIs were used to treat depression patients, the severity of the patient's condition should be fully evaluated and the behavioral tendency of the patient should be paid close attention to.

\section{Conflicts of Interest}

The authors declare no conflicts of interest regarding the publication of this paper.

\section{References}

Böhm, R., Von Hehn, L., Herdegen, T. et al. (2016). OpenVigil FDA-Inspection of U.S. 
American Adverse Drug Events Pharmacovigilance Data and Novel Clinical Applications. Plos One, 11, e157753. https://doi.org/10.1371/journal.pone.0157753

Friedman, R. A., \& Leon, A. C. (2007). Expanding the Black Box-Depression, Antidepressants, and the Risk of Suicide. New England Journal of Medicine, 356, 2343. https://doi.org/10.1056/NEJMp078015

Hammad, T. A., Laughren, T., \& Racoosin, J. (2006). Suicidality in Pediatric Patients Treated with Antidepressant Drugs. Archives of General Psychiatry, 63, 332-339. https://doi.org/10.1001/archpsyc.63.3.332

He, Z. Y., Zhu, W., \& Wu, S. (2018). Effect of Selective Serotonin Reuptake Inhibitors on Cognitive Function in Adolescent Patients with Depression. China Pharmaceuticals, 27, 43-46.

Leon, A. C. (2007). The Revised Warning for Antidepressants and Suicidality: Unveiling the Black Box of Statistical Analyses. American Journal of Psychiatry, 164, 1786-1789. https://doi.org/10.1176/appi.ajp.2007.07050775

Luo, L., Li, Q. L., \& Liu, J. G. (2008). Overview of Researches on Safety of Selective Serotion Inreuptake Inhibitors. Adverse Drug Reactions Journal, 10, 412-417.

Nardi, B., Francesconi, G., Catenadell'Osso, M. et al. (2013). Adolescent Depression: Clinical Features and Therapeutic Strategies. European Review for Medical \& Pharmacological Sciences, 17, 1546-1551.

Ng, W., Kennar, R., \& Uetrecht, J. (2014). Effect of Clozapine and Olanzapine on Neutrophil Kinetics: Implications for Drug-Induced Agranulocytosis. Chemical Research in Toxicology, 27, 1104-1108. https://doi.org/10.1021/tx500183x

Radovic, A., Farris, C., Reynolds, K. et al. (2014). Primary Care Providers' Initial Treatment Decisions and Antidepressant Prescribing for Adolescent Depression. Journal of Developmental \& Behavioral Pediatrics, 35, 28-37. https://doi.org/10.1097/DBP.0000000000000008

Rothman, K. J., Lanes, S., \& Sacks, S. T. (2004). The Reporting Odds Ratio and Its Advantages over the Proportional Reporting Ratio. Pharmacoepidemiology \& Drug Safety, 13, 519-523. https://doi.org/10.1002/pds.1001

Spindelegger, C. J., Papageorgiou, K., Grohmann, R. et al. (2015). Cardiovascular Adverse Reactions during Antidepressant Treatment: A Drug Surveillance Report of German-Speaking Countries between 1993 and 2010. International Journal of Neuropsychopharmacology, 18, u80. https://doi.org/10.1093/ijnp/pyu080

Wang, C. Y. (2011). Brief Description of Weight Gain Effect of Antidepressants. Sichuan Mental Health, 24, 125-126.

Wong, C. K., Ho, S. S., Saini, B. et al. (2015). Standardisation of the FAERS Database: A Systematic Approach to Manually Recoding Drug Name Variants. Pharmacoepidemiology \& Drug Safety, 24, 731-737. https://doi.org/10.1002/pds.3805

Yu, Y. (2016). Adverse Drug Reaction Knowledge Integration and Application Research Based on Big Data Mining. Jilin: Jilin University.

Zhou, M., Chen, M., \& Xu, Y. (2010). Retrospective Analysis of Adverse Reactions of Selective Serotonin Reuptake Inhibitors. Chinese Journal of Hospital Pharmacy, 30, 619-620.

Zhu, J. F. (2018). Adverse Reactions of Antidepressants. Herald of Medicine, 10, 1198-1202. 